\title{
QUALIDADE DE VIDA DOS CUIDADORES DE PACIENTES DEPENDENTES NO PROGRAMA DE SAÚDE DA FAMÍLIA ${ }^{1}$ QUALITY OF LIFE OF FAMILY CAREGIVERS OF PATIENTS DEPENDENT ON THE FAMILY HEALTH PROGRAM \\ LA CALIDAD DE VIDA DE LOS CUIDADORES DE ENFERMOS DEPENDIENTES EN EL PROGRAMA DE SALUD DE LA FAMILIA
}

\author{
Fernanda Amendolaㄹ, Maria Amélia de Campos Oliveira³ , Márcia Regina Martins Alvarenga
}

\footnotetext{
${ }^{1}$ Dissertação apresentada à Escola de Enfermagem da Universidade de São Paulo (EEUSP), no ano de 2007. Pesquisa financiada pela Fundação de Amparo à Pesquisa do Estado de São Paulo, Processo No 2007/00532-1.

2 Doutoranda do Programa de Pós-Graduação em Enfermagem pela EEUSP. Enfermeira do Programa Saúde da Família / Associação Congregação Santa Catarina. São Paulo, Brasil.

${ }^{3}$ Livre Docente. Professora Associada da EEUSP. São Paulo, Brasil.

${ }^{4}$ Doutoranda no Programa de Pós-Graduação em Enfermagem da EEUSP. São Paulo, Brasil.
}

PALAVRAS-CHAVE: Qualidade de vida. Cuidadores. Assistência domiciliar. Atenção primária à saúde. Programa Saúde da Família.

KEYWORDS: Quality of life. Caregivers. Home nursing. Primary health care. Family Health Program.
RESUMO: O estudo teve como objetivo avaliar a associação entre a percepção subjetiva da qualidade de vida dos cuidadores familiares de pacientes dependentes atendidos pelo Programa Saúde da Família e as características sociodemográficas e de saúde, grau de sobrecarga percebida e grau de independência funcional do paciente. Foram entrevistados 66 cuidadores, de dezembro 2006 a março de 2007, utilizando os instrumentos WHOQOL-bref; Zarit Burden Interview e a Medida de Independência Funcional para os pacientes. Houve o predomínio de mulheres cuidadoras, donas de casa e em média com 50,5 anos. A média da medida de independência funcional foi 57,82 classificando a amostra de pacientes em dependência modificada. A escala Zarit correlacionou-se negativamente com todos os domínios do WHOQOL-bref. As variáveis independentes estatisticamente relacionadas à qualidade de vida geral, no modelo múltiplo, foram: escala de sobrecarga, a presença de companheiro e a presença de doença no cuidador.

ABSTRACT: The objective of this study was to evaluate the association between the quality of life of family caregivers of dependent patients served by the Brazilian Family Health Program healthcare teams and socio-demographic characteristics, health condition, level of perceived burden, and degree of functional independence of the patient. Interviews were administered to 66 caregivers between December, 2006 and March, 2007, and utilizing the WHOQOL-bref; Zarit Burden Interview, and Functional Independence Measure of the patients as instruments. The caregivers were predominantly women, housewives, with an average age of 50.5 years. The average functional independence measure was 57.82, classifying the sample of patients in modified dependence. The Zarit was negatively correlated with all domains of the WHOQOL-bref. The statistically independent variables related to overall quality of life in the multiple model were: burden scale, the presence of a companion, and the presence of disease in the caregiver.
PALABRAS CLAVE: Calidad de vida. Cuidadores. Atención domiciliaria de salud. Atención primaria de salud. Programa salud de la familia.
RESUMEN: La investigación tuvo como objetivo evaluar la asociación entre la calidad de vida de los cuidadores familiares de enfermos dependientes atendidos por el Programa Salud de la Familia y las características sociales, demográficas, y de salud, así como el grado de sobrecarga percibido y el grado de independencia funcional del enfermo. Fueron entrevistados 66 cuidadores, en el período de diciembre 2006 a marzo 2007, utilizando un cuestionario de caracterización WHOQOL-bref, Zarit Burden Interview y la Medida de Independencia Funcional para los enfermos. Hubo el predominio de mujeres cuidadoras, amas de casa, con un promedio de 50,5 años de edad. La media de la medida de independencia funcional fue 57,82, clasificando los enfermos en dependencia modificada. La escala Zarit se correlacionó negativamente con todos los dominios del WHOQOL-bref. Las variables independientes estadísticamente relacionadas a la calidad de vida general, en el modelo múltiple, fueron: escala de sobrecarga, la presencia del compañero y la presencia de enfermedad en el cuidador.
Fernanda Amendola

Endereço: Departamento de Enfermagem em Saúde Coletiva da EEUSP

Av. Dr. Enéas de Carvalho Aguiar, 419

05.403-000 - Cerqueira César, São Paulo, SP, Brasil.

E-mail: famendola@usp.br
Artigo original: Pesquisa Recebido em: 15 de outubro de 2007 Aprovação final: 18 de abril de 2008 


\section{INTRODUÇÃO}

As mudanças nos perfis demográficos e de morbi-mortalidade da população mundial ao longo do último século e conseqüentes desafios para os sistemas de saúde explicam o crescente interesse dos pesquisadores pelo cuidado domiciliário à saúde, realizado no âmbito da família e envolvendo cuidadores leigos. Com a implementação do Programa Saúde da Família (PSF) na Atenção Básica $(\mathrm{AB})$, os cuidados domiciliários de saúde tornaram-se parte integrante das ações em saúde nesse nível de atenção. O cadastramento das famílias feito pelos agentes comunitários de saúde levou-os aos domicílios de usuários e tornou visíveis as necessidades de saúde de pacientes com perdas funcionais e dependência, antes confinados em seus lares, e de seus cuidadores.

As tarefas atribuídas ao cuidador, muitas vezes sem a orientação adequada, o suporte das instituições de saúde, a alteração das rotinas e o tempo despendido no cuidado têm impactos sobre a qualidade de vida do cuidador. É de se esperar que a qualidade de vida do cuidador familiar principal seja afetada negativamente por tais circunstâncias, o que é corroborado por vários estudos. Ainda assim, qualidade de vida e sobrecarga são construtos diferentes, e que, portanto, devem ser abordados com diferentes instrumentos de medida.

A qualidade de vida tornou-se um conceito amplamente difundido em diversas áreas, principalmente no meio acadêmico, por meio de estudos científicos relacionados à saúde. Alguns desses estudos têm sido criticados por autores que alegam que os instrumentos criados para medir a qualidade de vida na verdade avaliam diferentes fenômenos. Isto decorre da falta de consenso sobre o construto e da complexidade do fenômeno que se pretende avaliar. Há, contudo, concordância sobre o fato de que a qualidade de vida é composta por aspectos objetivos e subjetivos, positivos e negativos. As avaliações objetivas referem-se às conseqüências observáveis, enquanto as subjetivas dizem respeito à percepção ou avaliação pessoal sobre determinado aspecto abordado.

A avaliação da qualidade de vida dos cuidadores familiares deve contemplar tanto os aspectos positivos quanto os negativos, pois muitas situações consideradas negativas por meio de indicadores objetivos podem ser subjetivamente percebidas como positivas pelos indivíduos em questão. Tanto a dimensão objetiva quanto a subjetiva, é importante para o enriquecimento das informações coletadas com a finalidade de avaliar a qualidade de vida de cuidadores, permitindo melhor interpretar sua realidade de vida e saúde.

Nesta investigação, optou-se por utilizar o questionário de avaliação subjetiva de Qualidade de Vida (QV) desenvolvido pela Organização Mundial da Saúde (OMS), o World Health Organization Quality of Life instrument-bref (WHOQOL-bref), versão abreviada do World Health Organization Quality of Life instrument -100 itens (WHOQOL-100). Trata-se de um instrumento que adota um conceito multidimensional de QV , é amplamente utilizado no Brasil e em outros países e foi construído por meio de um levantamento sobre o que as pessoas consideram importante abordar ao medir QV.

O presente estudo buscou analisar a associação entre a percepção subjetiva da qualidade de vida de cuidadores familiares de pacientes com dependência atendidos pelo PSF e as características sociodemográficas, a condição de saúde dos cuidadores, as características do paciente e o escore da sobrecarga.

\section{MATERIAL E MÉTODO}

Trata-se de um estudo descritivo e transversal, no qual foram realizadas entrevistas domiciliares através de questionário estruturado e escalas padronizadas, a cuidadores e pacientes cadastrados e atendidos pelo Programa de Saúde da Família - Associação Congregação Santa Catarina (PSF-ACSC) no período de dezembro/2006 a março/2007.

As Unidades Básicas de Saúde do PSF-ACSC situam-se na zona sul da capital paulista na Subprefeitura da Cidade Ademar. É uma região com população total de aproximadamente 370.797 habitantes, composta predominante por pessoas com baixo poder aquisitivo.

A amostra foi composta por 66 cuidadores familiares de pacientes dependentes, atendida por equipes de Saúde da Família distribuídas entre sete Unidades Básicas de Saúde. Definiu-se cuidador a pessoa que assume o papel de assistir ao familiar em situação de dependência, que necessita de ajuda no desempenho de suas atividades da vida diária, como alimentação, locomoção, banho entre outros.

Os critérios de inclusão para seleção dos sujeitos foram: familiares com idade igual ou maior de 16 anos, não remunerados e prestando cuidados há mais de dois meses. Foram excluídas as famílias na qual não foi possível identificar o cuidador principal. 
Obteve-se aprovação dos Comitês de Ética em Pesquisa da Escola de Enfermagem da Universidade de São Paulo ( $n^{\circ}$ 488-2005), da Secretaria Municipal de Saúde de São Paulo (nº 041/06) e parecer favorável do PSF-ACSC. Os participantes, depois de informados dos objetivos da pesquisa, assinaram o Termo de Consentimento Livre e Esclarecido. Esta pesquisa foi financiada pela Fundação de Amparo à Pesquisa do Estado de São Paulo (Processo n ${ }^{\circ}$ 2007/00532-1).

O grau de dependência do paciente foi determinado pela Medida de Independência Funcional (MIF), ${ }^{1}$ que também verificou sua reprodutibilidade. O instrumento é composto por 18 categorias agrupadas em seis dimensões: autocuidado, controle de esfíncteres, transferências, locomoção, comunicação e cognição social. Cada item pontua de 1 (dependência total) a 7 (independência completa). Obtém-se escore total mínimo de 18 e máximo de 126 pontos que caracterizam os níveis de dependência.

Para mensurar a sobrecarga dos cuidadores utilizou-se a escala Zarit Burden Interview (ZBI), ${ }^{2}$ composto por 22 itens que avaliam a relação cuidadorpaciente, a condição de saúde, o bem-estar psicológi$\mathrm{co}$, finanças e vida social. A escala de respostas varia de 0 a 4, de acordo com presença ou intensidade de uma resposta afirmativa $(0=$ nunca, $1=$ raramente, $2=$ algumas vezes, 3 =freqüentemente e $4=$ sempre). A exceção é o último item, no qual o entrevistado é questionado se está se sentindo sobrecarregado no papel de cuidador e as respostas são: $0=$ nem um pouco, $1=$ um pouco, $2=$ moderadamente, $3=$ muito, $4=$ extremamente. O escore total da escala varia de 0 a 88. Quanto maior o escore maior a sobrecarga.

O questionário utilizado para avaliar a dos cuidadores, o WHOQOL-bref, ${ }^{3}$ contém 26 questões sendo duas gerais (qualidade de vida geral e satisfação com a saúde) e 24 englobadas nos domínios Físico, Psicológico, Relações Sociais e Meio Ambiente. As duas questões gerais são calculadas em conjunto para gerar um único escore independente dos outros escores dos domínios, no qual denominamos de Índice Geral de Qualidade de Vida (IGQV).

Em estudo semelhante, ${ }^{4}$ a maioria dos coeficientes de correlação entre as características sociodemográficas e de saúde e os escores do instrumento de qualidade de vida e seus domínios foram superiores a 0,35. Com base nesse achado e assumindo um erro do tipo I de $5 \%$ e um poder de $80 \%$, estimou-se que seriam necessários pelo menos 62 cuidadores.
O tratamento dos dados foi feito utilizandose o Software Statistical Package for the Social Sciences 12.0. Para avaliar a correlação das variáveis quantitativas do cuidador e do paciente com a QV do cuidador utilizou-se o coeficiente de correlação de Spearman. Também foi estimado um modelo de regressão linear múltipla, tendo como variável dependente o escore do IGQV e, como variáveis independentes, as características sociodemográficas, a condição de saúde dos cuidadores, as características do paciente e o escore de sobrecarga. Para este estudo elegeu-se apenas o escore do IGQV, por considerá-lo um indicador mais abrangente e adequado aos objetivos do estudo.

Foram selecionadas para o processo de modelagem múltipla aquelas variáveis com p<0,20. O processo de seleção foi o stepwise forward e a variável independente permaneceu no modelo múltiplo quando $p<0,05$. O nível de significância adotado para o estudo foi de $5 \%$.

\section{RESULTADOS}

A maioria dos pacientes cuidados era do sexo feminino $(57,6 \%)$ e com média de 63,45 anos $(\mathrm{dp}=22,5)$. A patologia dos pacientes mais citada pelos cuidadores foi acidente vascular encefálico $(39,4 \%)$. A média total da escala MIF foi de 57,82 $(d p=30,28)$ e a mediana de 53,0 , classificando a amostra em dependência modificada. As pontuações mais baixas foram encontradas nos domínios autocuidado $(17,45)$, transferência $(8,73)$ e locomoção $(4,76)$. O valor de alpha de Cronbach de cada domínio e da escala total foi bastante satisfatório em todos os domínios (acima de 0,70).

Os cuidadores eram, em sua maioria, mulheres $(83,3 \%)$, filhas(os) $(37,9 \%)$, casadas ou em união consensual $(62,2 \%)$ com média de idade de 50,5 anos ( $d p=14,84$; mediana de 51 anos) e referiram ter alguma doença $(63,6 \%)$. As principais características estão descritas na Tabela 1.

Entre os cuidadores predominou o diagnóstico de hipertensão arterial (39,4\%), 13,6\% faziam uso de medicamentos para depressão ou calmantes, $54,5 \%$ referiram algum tipo de dor e dormiam em média sete horas por dia.

O tempo como cuidador variou de três meses a 42 anos, com uma média de 9,8 anos cuidando de seu familiar ( $d p=9,6$ anos) e mediana de 6 anos. A renda per capita média foi de $\mathrm{R} \$ 273,17^{*}$ $(d p=161,38)$ e mediana de $R \$ 233,33$.

* Salário Mínimo (SM) no período de R\$350,00 
Tabela 1 - Características dos cuidadores familiares. São Paulo - SP, 2007.

\begin{tabular}{lc}
\hline Variável & $\begin{array}{c}\text { Cuidadores } \\
\text { ( } \mathbf{n}=66)\end{array}$ \\
\hline Sexo (\%) & \\
Feminino & 83,3 \\
Masculino & 16,7 \\
Idade (\%) & \\
Média & 50,5 \\
dp & 14,8 \\
Mínimo & 17 \\
Máximo & 85 \\
Escolaridade (\%) & \\
Analfabeto & 21,2 \\
Ensino fundamental incompleto & 35,0 \\
Ensino fundamental completo ou mais & 43,8 \\
Estado marital (\%) & \\
Casados / união consensual & 62,2 \\
Solteiros & 22,7 \\
Parentesco com relação ao paciente (\%) \\
Filha/filho \\
Marido/esposa \\
Pai/mãe \\
Trabalho (\%) \\
Exercendo atividade & 37,9 \\
Dona de casa & 24,2 \\
Aposentado ou pensionista & 12,1 \\
Presença de morbidade (\%) & \\
Sim & 25,8 \\
Não & 43,9 \\
Atividade social (\%) & 18,2 \\
Religião & \\
Visitar parentes / amigos & \\
Não têm atividades & 63,6 \\
De quem recebe ajuda (\%) & 36,4 \\
Parentes & \\
Ninguém & 39,0 \\
& 14,2 \\
\hline & 35,1 \\
& 58,5 \\
& 27,1 \\
\hline
\end{tabular}

A escala de avaliação da sobrecarga ZBI variou de 0 a 67 , com média de 32,12 (dp=14,7). O alpha de Cronbach para o ZBI foi de 0,86, demonstrando bom índice de consistência interna para esta amostra.

No WHOQOL-bref, os domínios Físico e Relações Sociais apresentaram os maiores escores (66,72 e 60,86, respectivamente), enquanto que o domínio Meio Ambiente apresentou o menor $(52,51)$. A confiabilidade do instrumento, avaliada pelo alpha de Cronbach, mostrou bom índice de consistência interna nos domínios Físico e Psicológico $(0,82$ e 0,74 , respectivamente) e baixo nos domínios Relações Sociais e Meio Ambiente $(0,49$ e 0,45 , respectivamente).

A Tabela 2 mostra a correlação da QV do cuidador com as variáveis quantitativas do cuidador e do paciente, através da correlação de Spearman. Destaca-se a correlação negativa estatisticamente significativa no domínio Físico com a idade do cuidador e a idade do paciente e no IGQV e todos os demais domínios com a escala Zarit, indicando que quanto menor o escore médio do IGQV e dos demais domínios maior o escore médio do Zarit, portanto quanto maior a sobrecarga menor os escores de QV.

A Tabela 3 apresenta o modelo de regressão linear múltipla final. As variáveis independentes estatisticamente relacionadas com o IGQV foram a escala de Zarit $(\beta=-0,542 ; p=0,001)$, ter companheiro(a) $(\beta=+10,355 ; \mathrm{p}=0,039)$ e a presença de doença no cuidador $(\beta=-11,701 ; \mathrm{p}=0,021)$. Conclui-se que para cada ponto do Zarit, há, em média, uma diminuição de 0,542 no IGQV.

Tabela 2 - Coeficientes de correlação de Spearman $\left(\mathrm{r}_{\mathrm{sp}}\right)$ entre os domínios WHOQOL-bref e as demais variáveis. São Paulo - SP, 2007.

\begin{tabular}{|c|c|c|c|c|c|c|c|}
\hline $\begin{array}{c}\text { Domínios } \\
\text { WHOQOL-bref }\end{array}$ & $\begin{array}{c}\text { Idade } \\
\text { paciente } \\
r_{\text {sp }} \\
(p)\end{array}$ & $\begin{array}{l}\text { Idade } \\
\text { cuidador } \\
r_{\mathrm{sp}} \\
\text { (p) }\end{array}$ & $\begin{array}{l}\text { Renda per } \\
\text { capita } \\
r_{\mathrm{sp}} \\
\text { (p) }\end{array}$ & $\begin{array}{c}\text { № de pessoas que } 7 \\
\text { mora no domicílio } \\
r_{\mathrm{sp}} \\
\text { (p) }\end{array}$ & $\begin{array}{l}\text { Tempo como } \\
\text { cuidador } \\
r_{\mathrm{sp}} \\
(\mathbf{p})\end{array}$ & $\begin{array}{l}\text { Zarit } \\
r_{\text {sp }} \\
(p)\end{array}$ & $\begin{array}{l}\text { MIF total } \\
r_{\text {sp }} \\
\text { (p) }\end{array}$ \\
\hline QV geral & $\begin{array}{c}-0,10 \\
(0,420)\end{array}$ & $\begin{array}{c}-0,07 \\
(0,565)\end{array}$ & $\begin{array}{c}0,20 \\
(0,113)\end{array}$ & $\begin{array}{c}-0,04 \\
(0,746)\end{array}$ & $\begin{array}{c}0,19 \\
(0,134)\end{array}$ & $\begin{array}{l}-0,35^{*} \\
(0,005)\end{array}$ & $\begin{array}{c}0,03 \\
(0,831)\end{array}$ \\
\hline Físico & $\begin{array}{l}-0,28^{*} \\
(0,023)\end{array}$ & $\begin{array}{c}-0,47^{\star} \\
(<0,001)\end{array}$ & $\begin{array}{c}0,08 \\
(0,517)\end{array}$ & $\begin{array}{c}0,12 \\
(0,359)\end{array}$ & $\begin{array}{c}-0,12 \\
(0,352)\end{array}$ & $\begin{array}{l}-0,29^{*} \\
(0,018)\end{array}$ & $\begin{array}{c}0,22 \\
(0,081)\end{array}$ \\
\hline Psicológico & $\begin{array}{c}-0,07 \\
(0,587)\end{array}$ & $\begin{array}{c}-0,06 \\
(0,660)\end{array}$ & $\begin{array}{c}0,18 \\
(0,168)\end{array}$ & $\begin{array}{l}0,07 \\
0,580\end{array}$ & $\begin{array}{c}0,15 \\
0,246\end{array}$ & $\begin{array}{l}-0,39^{\star} \\
0,001\end{array}$ & $\begin{array}{c}0,05 \\
0,691\end{array}$ \\
\hline Relações sociais & $\begin{array}{c}-0,21 \\
(0,096)\end{array}$ & $\begin{array}{c}-0,16 \\
(0,187)\end{array}$ & $\begin{array}{c}0,09 \\
(0,466)\end{array}$ & $\begin{array}{c}0,30^{*} \\
(0,014)\end{array}$ & $\begin{array}{c}0,20 \\
(0,103)\end{array}$ & $\begin{array}{l}-0,26^{*} \\
(0,033)\end{array}$ & $\begin{array}{c}-0,09 \\
(0,485)\end{array}$ \\
\hline Meio ambiente & $\begin{array}{l}-0,32^{*} \\
(0,008)\end{array}$ & $\begin{array}{c}0,03 \\
(0,793)\end{array}$ & $\begin{array}{c}0,06 \\
(0,649)\end{array}$ & $\begin{array}{c}0,04 \\
(0,741)\end{array}$ & $\begin{array}{c}0,26^{*} \\
(0,038)\end{array}$ & $\begin{array}{l}-0,35^{\star} \\
(0,004)\end{array}$ & $\begin{array}{c}0,11 \\
(0,382)\end{array}$ \\
\hline
\end{tabular}

* correlação estatisticamente significativa $(p<0,05)$ 
O cuidador casado ou em união consensual (com companheiro) tem, em média, mais 10,355 pontos no IGQV. O cuidador que apresenta alguma doença tem, em média, menos 11,701 pontos no IGQV quando comparado àquele que não refere doenças. Feita a análise de resíduos, verificou-se que, do ponto de vista estatístico, o modelo final não apresenta viés.

Tabela 3 - Modelo de regressão linear múltiplo final para o IGQV. São Paulo - SP, 2007.

\begin{tabular}{lcc}
\hline Variável & $\begin{array}{c}\text { Coeficiente de } \\
\text { regressão } \boldsymbol{\beta}\end{array}$ & $\mathbf{p}$ \\
\hline Zarit & $-0,542$ & 0,001 \\
Com companheiro(a) & $+10,355$ & 0,039 \\
Presença de doença & $-11,701$ & 0,021 \\
\hline
\end{tabular}

$\mathrm{r}^{2}$ ajustado $=0,23 ; \quad \mathrm{p}$ (modelo) $<0,001$

\section{DISCUSSÃO}

Elegemos a QV como a variável dependente do estudo e os outros dados como variáveis independentes, buscando correlacionar as variáveis exploradas tanto relativas aos cuidadores quanto aos pacientes com o grau de implicação na QV.

Os cuidadores familiares, objeto desse estudo, foram recortados pelo nível e modelo de atenção aos quais esses sujeitos estão inseridos. Poucos estudos avaliam os cuidadores na $A B,{ }^{5-6}$ sendo preponderantes os que avaliam o cuidador em função da doença do paciente ${ }^{7-8}$ ou por serviços vinculados a hospitais. ${ }^{9-10}$

A maioria dos pacientes, cuidado por seus familiares, era do sexo feminino e idoso. A média de pontuação da MIF mostrou que os pacientes atendidos pelas equipes de saúde da família, em média, conseguem realizar as tarefas básicas da vida diária em até $50 \%$, ou seja a maioria dos pacientes apresenta necessidades de cuidado compatíveis com a $\mathrm{AB}$, entretanto, essas pessoas não conseguem realizar atividades da vida diária sem a ajuda de um cuidador.

O predomínio de mulheres cuidadoras, casadas, donas de casa e em média com 50,5 anos, também são características freqüentes encontradas em diversos estudos seja no âmbito nacional ou internacional. ${ }^{9,11-13}$ Os achados reforçam o papel social da mulher, historicamente determinado, onde é esperado que ela seja a cuidadora principal e que o cuidado a pessoas dependentes fique a cargo dos parentes mais próximos. A escolaridade é predominantemente baixa em diversos estudos com cuidadores. ${ }^{6,9,12} \mathrm{O}$ baixo nível pode contribuir para o papel de cuidador ser atribuído a essas pessoas, na medida em que se sabe que a inserção no mercado de trabalho formal é mais difícil àqueles indivíduos com baixa escolaridade. Assim, é mais provável que essas pessoas se dediquem aos serviços domésticos e a tarefa de cuidar do familiar dependente como uma extensão dessa atividade.

O índice médio de sobrecarga do cuidador, avaliado através do ZBI foi de 32,12 pontos, portanto, estavam moderadamente sobrecarregados. Estudos que utilizaram essa escala, apresentaram dados semelhantes, indicando escores de moderado a alto. ${ }^{10,14-15}$

A idade do paciente mostrou correlação com o domínio Físico, indicando que, quanto maior a idade, menor o escore médio nesse domínio, já que pacientes mais velhos requerem cuidados que sobrecarregam fisicamente o cuidador. A idade do paciente também se correlacionou com o domínio Meio Ambiente, ou seja, quanto maior a idade, menor o escore do domínio. O paciente idoso normalmente demanda condições específicas para o seu cuidado devido à diminuição de algumas capacidades ao longo dos anos. Talvez por isso os cuidadores de pacientes mais velhos estejam menos satisfeitos com o ambiente físico, condições de moradia, recursos financeiros, serviços de saúde e transporte, itens relativos ao domínio Meio Ambiente. A idade do cuidador parece ser uma variável importante na avaliação da qualidade de vida do cuidador. ${ }^{13,15}$ No presente estudo, quanto maior a idade do cuidador, menor o escore no domínio Físico. Em estudo com cuidadores de pacientes com esquizofrenia, ${ }^{15}$ constatou-se que quanto maior a idade, maior a sobrecarga percebida do cuidador. Cuidadores mais velhos parecem mais susceptíveis a sobrecarga, porém os mais jovens podem sofrer mais isolamento e maiores restrições sociais, proporcionais às maiores possibilidades de atividades de lazer e sociais de sua faixa etária. ${ }^{13}$ Foi encontrada correlação positiva entre o número de pessoas que moram no mesmo domicílio e o domínio Relações Sociais. O número de pessoas que residem no mesmo domicílio parece significar também uma melhor rede de apoio, visto que há mais pessoas próximas que podem ajudar e se revezar no cuidado.

Os cuidadores que referiram maior sobrecarga avaliaram menos satisfatoriamente sua saúde e qualidade de vida, o que demonstra que a aplicação de um instrumento específico para as atividades do cuidador correlacionou-se satisfatoria- 
mente com o instrumento de avaliação subjetiva de qualidade de vida geral, o WHOQOL-bref. Além disso, mostrou ser um bom indicador da situação do cuidador, podendo fornecer subsídios para o planejamento de ações e intervenções destinadas àqueles que apresentam maior sobrecarga e que, portanto, tem a QV mais comprometida. Portanto, verificou-se que indivíduos mais sobrecarregados pela tarefa de cuidar apresentam diminuição na percepção de sua qualidade de vida.

A presença de um companheiro, que pode ser o próprio paciente ou não, pode trazer benefícios com relação à menor número de sentimentos de solidão e maior apoio. Muitos são os relatos de companheiros que deixaram suas esposas ao ter de receber em suas casas pais ou parente das mesmas, que já não podiam viver sozinhos e precisaram então recorrer às casas de seus filhos ou outros parentes. Nesta amostra, a presença de companheiro pareceu favorável ao bem-estar dos cuidadores, o que necessariamente pode não ocorrer ou até ocorrer o contrário, ou seja, a presença do companheiro pode ser um fator estressor para o cuidador. A doença também se apresentou como preditor para menor satisfação com a saúde e a QV geral do cuidador. Algumas doenças provocam uma série de sinais e sintomas que podem interferir na avaliação do cuidador de sua vida e saúde. Concomitantemente a propostas de intervenções voltadas para a saúde dos cuidadores, as equipes de saúde devem avaliar o grau de implicação das enfermidades existentes e como essas podem interferir na tarefa de cuidar e na sua qualidade de vida.

\section{CONSIDERAÇÕES FINAIS}

O estudo indicou que cuidadores familiares de pacientes dependentes estão cuidando de pessoas com alto grau de incapacidade funcional e que a sobrecarga afeta diretamente a QV desses familiares. Somando-se a isso, trata-se de uma população carente social e economicamente, com pouca oferta de área de lazer e cultura, com baixo nível de escolaridade, acometidas por doenças crônicas e contam quase que, exclusivamente, com a rede de apoio informal familiar.

Além das ações em âmbito particular e pontual como orientações de como realizar o cuidado, assistência à saúde, apoio físico e emocional aos cuidadores, cabe às equipes de saúde da família ações que contemple os princípios fundamentais do Sistema Único de Saúde, possibilitando uma intervenção mais efetiva na mudança do perfil de saúde e doença dessa população. Isso significa atuações intersetoriais e com a participação da comunidade para planejarem as ações que busquem melhorar a qualidade de vida das pessoas dependentes e seus cuidadores. Para tanto, propomos - Investir e melhorar a estrutura social com a participação da comunidade ("empoderamento") com o objetivo de discutir como melhorar a qualidade de vida; - Identificar os recursos da comunidade (suporte social) para propor medidas alternativas para os cuidados domiciliários, com a possibilidade de criar serviços piloto, como centros-dia, com o objetivo de melhorar a qualidade de vida de pacientes e dos cuidadores; - Utilizar de forma efetiva dos instrumentos de avaliação descritos nos cadernos de AB do próprio Ministério da Saúde para identificar os pacientes dependentes funcionalmente e avaliar seu grau de comprometimento, assim como identificar a possível sobrecarga do cuidador.

Neste estudo identificamos algumas variáveis que podem influenciar na avaliação da QV dos cuidadores. Os dados obtidos, através de comprovação estatística significativa nos dão subsídios para identificarmos fatores de risco associados à pior percepção de qualidade de vida. A escala de sobrecarga, a presença de companheiro e a presença de doença mostraram ser preditores na percepção da qualidade de vida geral e da satisfação com a saúde dos cuidadores.

\section{REFERÊNCIAS}

1 Riberto M, Miyazaki MH, Jorge D, Sakamato H, Battistella LR. Reprodutibilidade da versão brasileira da Medida de Independência Funcional. Acta Fisiátrica. 2001 Abr; 8 (1): 45-52.

2 Scavufca M. Versão brasileira da escala Burden Interview para avaliação de sobrecarga em cuidadores de indivíduos com doenças mentais. Rev. Bras. Psiquiatr. 2002 Mar; 24 (1): 12-7.

3 Fleck MPA, Lousada S, Xavier M, Chachamovich E, Vieira G, Santos L, et al. Aplicação da versão em português do instrumento abreviado de avaliação da qualidade de vida "WHOQOL-bref". Rev. Saúde Pública 2000 Abr; 34 (2): 178-83.

4 Peñaranda AP, Ortiz LG, Góngora JN. El cuidador primário de familiares con dependência: calidad de vida, apoyo social y salud mental [tese]. Salamanca (ES): Universidad de Salamanca/Facultad de Medicina; 2006.

5 Watanabe HAW, Derntl AM. Cuidadores de idosos: uma experiência em Unidade Básica de Saúde Projeto CapacIDADE. Mundo Saúde 2005 Out/Dez; 29 (4): 639-44.

6 Garcia MAA, Frigerio RM, Miyamoto DA, Merlin 
SS. Idosos e cuidadores fragilizados? Mundo Saúde. 2005 Out/Dez; 29 (4): 645-52.

7 Schestatsky P, Zanatto VC, Margis R, Chachamovich E, Reche M, Batista RG, et al. Quality of life in a brazilian sample of patients with parkinson's disease and their caregivers. Rev. Bras. Psiquiatr. 2006 Set; 28 (3): 209-11.

8 Brouwer WBF, Exel NJA, Berg B, Dinant HJ, Koopmanschap MA, Bos GAM. Burden of caregiving: evidence of objective burden, subjective burden, and quality of life impacts on informal caregivers of patients with rheumatoid arthritis. Arthritis Rheumatism. 2004 Aug; 51 (4): 570-7.

9 Sportello EF. Caracterização das formas de vida e trabalho das cuidadoras familiares do Programa de Assistência Domiciliária do Hospital Universitário da Universidade de São Paulo [dissertação]. São Paulo (SP): USP/Escola de Enfermagem; 2003.

10 Garrido R, Menezes PR. Impacto em cuidadores de idosos com demência em um serviço psicogeriátrico. Rev. Saúde Pública. 2004 Dez; 38 (6): 835-41.

11 Karsch UMS. Envelhecimento com dependência: revelando cuidadores. São Paulo (SP): Educ; 1998.

12 Rodriguez PR. El apoyo informal a las personas mayors en España y la protección social a la dependencia. Del facilismo a los derechos de ciudadanía. Rev. Esp. Geriatr. Gerontol. 2005 Dec; 40 (supl. 3): 5-15.

13 Glozman JM. Quality of life caregivers. Neuropsychology rev. 2004 Dec; 14 (4): 183-96.

14 Llach XBL, Suriñach NL, Gamisans MR. Calidad de vida, tiempo de dedicación y carga percibida por el cuidador principal informal del enfermo de Alzheimer. Aten Primária 2004 Sept; 34 (4): 170-7.

15 Urizar CA, Maldonado JG. Burden of care in families of patients with schizophrenia. Qual. life Res. 2006 May; 15 (4): 719-24. 